\title{
United States Pharmacopeia
}

National Cancer Institute

\section{Source}

National Cancer Institute. United States Pharmacopeia. NCI Thesaurus. Code C54397.

The official public standards-setting authority for all prescription and over-the-counter medicines, dietary supplements, and other healthcare products manufactured and sold in the United States. USP sets standards for the quality of these products and works with healthcare providers to help them reach the standards. USP promotes the public health by developing and disseminating quality standards and information for medicines, healthcare delivery, and related products and practices. USP is an independent, sciencebased public health organization. 